\title{
Correction to: Resistance of Arctic phytoplankton to ocean acidification and enhanced irradiance
}

\author{
C. J. M. Hoppe ${ }^{1,2}$ (1) $\cdot$ N. Schuback ${ }^{1,3} \cdot$ D. Semeniuk ${ }^{1} \cdot$ K. Giesbrecht ${ }^{4} \cdot$ J. Mol $^{5} \cdot$ H. Thomas ${ }^{5} \cdot$ M. T. Maldonado ${ }^{1}$. \\ B. Rost $^{2} \cdot$ D. E. Varela ${ }^{4,6} \cdot$ P. D. Tortell ${ }^{1,7,8}$
}

Published online: 2 February 2018

○) Springer-Verlag GmbH Germany, part of Springer Nature 2018

\section{Correction to: Polar Biol \\ https://doi.org/10.1007/s00300-017-2186-0}

The authors regret an error in the published article, where incorrect data was used to produce Figure 2, showing the temporal development of $\mathrm{pH}$ over the duration of the experiment. The corrected Fig. 2 shows that the error did not affect the interpretation of nor the conclusions drawn from the present dataset. The original article has been corrected.

The original article can be found online at https://doi.org/10.1007/ s00300-017-2186-0.

\section{J. M. Hoppe}

Clara.Hoppe@awi.de

1 Department of Earth, Ocean and Atmospheric Sciences, University of British Columbia, Vancouver, BC, Canada

2 Marine Biogeosciences, Alfred Wegener Institute Helmholtz Centre for Polar and Marine Research, Am Handelshafen 12, 27570 Bremerhaven, Germany

3 Department of Physics and Astronomy, Curtin University, Perth, WA, Australia

4 School of Earth and Ocean Sciences, University of Victoria, Victoria, BC, Canada

5 Department of Oceanography, Dalhousie University, Halifax, NS, Canada

6 Department of Biology, University of Victoria, Victoria, BC, Canada

7 Department of Botany, University of British Columbia, Victoria, BC, Canada

8 Peter Wall Institute for Advanced Studies, University of British Columbia, Vancouver, Canada 
Fig. 2 Time-course of seawater $\mathrm{pH}$ in incubation bottles for the LL LC (black circles), LL HC (black squares), HL LC (gray triangles) and HL HC (gray diamonds) treatments over the course of the experiment $(n=3$, $\pm 1 \mathrm{SD})$. $\mathrm{pH}$ was measured on the total scale

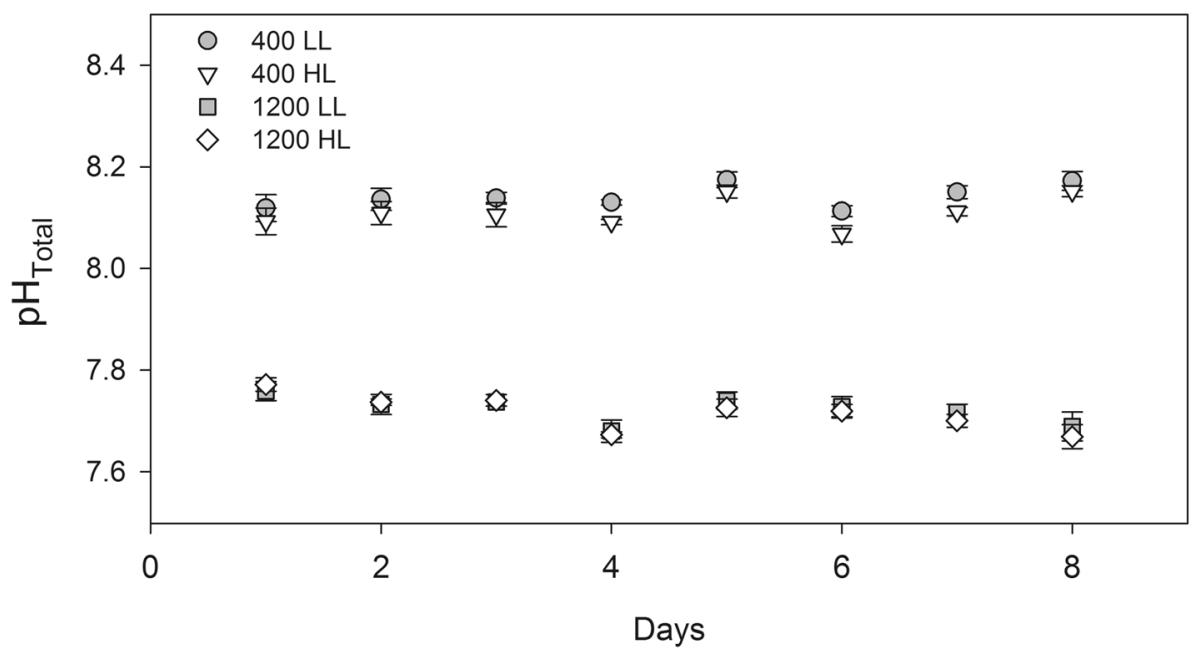

